\title{
Contributions of Psychopathological and Interpersonal Variables to Problematic Facebook Use in Adolescents and Young Adults
}

\author{
Henri Chabrol, ${ }^{1}$ Stephanie Laconi, ${ }^{1,}{ }^{*}$ Manon Delfour, ${ }^{1}$ and Axelle Moreau ${ }^{1}$ \\ ${ }^{1}$ Centre d'Etudes et de Recherche en PsychoPathologie (CERPP), University Toulouse 2, Toulouse, France \\ "Corresponding author: Stephanie Laconi, Centre d'Etudes et de Recherche en PsychoPathologie (CERPP), University Toulouse 2, Toulouse, France. Tel: +33-629441868, Fax: \\ +33-561503992, E-mail: stephanielaconi.b@gmail.com
}

Received 2015 August 29; Revised 2015 November 09; Accepted 2015 November 23.

\begin{abstract}
Background: Problematic Facebook use, also known as Facebook addiction, has recently been recognized as a cause of potential harm to adolescents and young adults. Some problematic Internet use risk factors have been linked to Facebook use. Yet few studies have explored the risk factors for problematic Facebook use in young people.

Objectives: The aim of this study was to evaluate the contribution of psychopathological variables, in particular borderline traits and interpersonal variables (i.e., parent and peer attachments and parental bonding styles), to the explanation of problematic Facebook use symptoms by taking gender into account and controlling for motives, one of the most important risk factors for problematic Facebook use.

Patients and Methods: The final sample consisted of 456 Facebook users (227 women) aged from 12 to 25 ( $M=20.5 ; S D=2.5$ ). These participants completed online self-report questionnaires assessing problematic Facebook use, motives for Facebook use, depressive symptoms, social anxiety, sensation seeking, borderline personality traits, parental bonding and attachment, and peer attachment. Results: Multiple regression analyses showed that motives and maternal overprotection were the main predictors of problematic Facebook use symptoms in both genders. Mediation analyses suggested that borderline personality traits and overprotection played different roles in the development of problematic Facebook use symptoms. In females, borderline traits were a mediator in the relation between maternal overprotection and problematic Facebook use symptoms. In males, maternal overprotection was a mediator in the relation between borderline traits and problematic Facebook use symptoms. To our knowledge, this is the first study exploring the contribution of parental bonding and borderline traits to problematic Facebook use.
\end{abstract}

Keywords: Social Media, Internet, Borderline Personality Disorder, Gender

\section{Background}

In recent years, problematic Facebook use (PFU), also called Facebook addiction, has emerged as a potential detriment to the health of adolescents and young adults $(1,2)$. However, few studies of PFU have been conducted in adolescents and students. Among university students, the approximate rate of PFU has been found to range between $2 \%$ and $10 \%$ (3-5). According to Andreassen, PFU tends to occur more regularly among younger than older users, and is more prevalent in women than men (6).

Several predictive factors of adolescent and young adult problematic internet use (PIU) (7-9) have also been linked to Facebook use. According to studies of PIU, motives play an important role by explaining 60\% of Facebook use variance among university students (10). Among college students, weekly time commitment, social motives, severe depression, anxiety, and insomnia also positively predicted PFU (2), as did self-inferiority and having a depressive character among university students (11). Socially anxious students tended to spend more time on Facebook, probably because online communication appears easier than a real face-to-face interaction, but also reported fewer Facebook friends $(1,10)$. Sensation seeking was found to be higher among Facebook users than non-users (10).

Among other personality characteristics, most studies have focused on the five-factor model of personality $(1,12)$. Personality disorders have not been sufficiently explored in PIU and PFU. However, borderline traits appear to be particularly related to time spent online and PIU among adults (13) and are a significant predictor of PFU among younger users (14). Borderline traits include a high need for social relationship and an intolerance of loneliness, which could explain the relationship with PFU.

Attachment styles have also been identified as potential predictors for Facebook use among university students (12) and adults (15) and were related to PFU among adolescents and young adults (5). It should be noted that borderline traits can be related to parental bonding and attachment. While adolescents with borderline traits experience more overprotection from their mothers (16), overprotection may increase the risk of borderline traits in adoles- 
cents. Conversely, borderline features may provoke overprotection, suggesting that these influences may be reciprocal (17). One previous study showed that participants with the highest scores for borderline traits had lower scores for functional relationships and higher scores for PFU (5), highlighting the need to explore the relationship between these variables and PFU.

\section{Objectives}

The principal aim of this study was to evaluate the contribution of psychopathological variables, in particular borderline traits, and interpersonal variables (i.e., parent and peer attachments and parental bonding styles) to PFU, controlling for one of the most important risk factors (i.e., motives) in a sample of adolescents and young adults. We hypothesized that mediations may contribute to explaining the relationships between maternal overprotection, borderline traits, and Facebook use.

\section{Patients and Methods}

\subsection{Participants and Procedure}

The sample comprised 456 Facebook users (348 women, 76\%), aged between 13 and 25 years old (mean age $\pm \mathrm{SD}=20.5 \pm 2.5$ ). They consisted of 328 university students (72\%), 52 employees (11\%), 32 inactive (7\%), 28 high-school students (6\%), 11 schoolboys (2\%), and 5 participants who did not indicate their social status (1\%). Only participants with a Facebook account were included in this study.

Participants were recruited via online system between November 2012 and February 2013 through Facebook, email, and forums. All participants were informed of the confidentiality and the anonymity of their responses, and agreed to give their free and informed consent. This study has been performed in accordance with the 1964 Declaration of Helsinki and its later amendments. This sample was previously $(5,14)$.

\subsection{Measures}

\subsubsection{Facebook Use and Motives}

The Internet Addiction Test (IAT) $(18,19)$, the most used and validated tool to assess PIU (20), has been adapted to assess PFU $(3,21)$. The 20 items are scored on a 5-point scale ranging from rarely to always (e.g., How often do you try to cut down the amount of time you spend on Facebook and fail?). The total score varies from 20 to 100, distinguishing normal (20 - 49), moderate problematic (50 - 79), and severe problematic uses (80 -100). Given the large overlap between PFU and PIU, and similarly to previous studies using a Facebook version of the IAT, participants scoring higher than 49 were considered problematic Facebook users (14, 21). The IAT generally presents good psychometric properties, with Cronbach's $\alpha$ ranged from $\alpha=0.63$ to $\alpha=0.93$ (20).

Motives for Facebook use were evaluated using the questionnaire of Facebook use motives (10). It is composed of 26 items exploring six motives (i.e., relationship maintenance, passing time, virtual community, entertainment, coolness, companionship). Each item is rated on a 5-point scale between exactly and not at all (e.g., to see other people's pictures). The total score varies from 26 to 130. Cronbach's $\alpha$ for each subscale are ranged between $\alpha=0.76$ and $\alpha=0.90$.

\subsubsection{Psychopathological Variables}

We assessed depressive symptoms using the short version of the Center for Epidemiologic Studies-Depression scale (CES-D-10) (22). Its 10 items assess the presence of depressive symptoms during the past week on a 4-point scale ranged from rarely or never to most of the time or all the time (e.g., I felt depressed). Scores vary from 0 to 30, with a score greater than 10 indicating the presence of significant depressive symptoms. Internal consistency of the CESD-10 is $\operatorname{good}(\alpha=0.75)$ (23).

Social anxiety was evaluated with the Social Anxiety Scale for Adolescents (SAS-A) $(24,25)$. It is composed of 18 items rated on a 5-point Likert scale ranged from not at all to all the time. The SAS-A is divided into three subscales: fear of negative evaluation (8 items), specific social avoidance of new or unfamiliar situations (6 items) and social avoidance and general distress ( 4 items) (e.g., I worry what others say about me). Total scores vary from 18 to 90 , with a 50-point cutoff score suggesting social anxiety. Cronbach's alphas of each subscale are respectively $\alpha=0.89, \alpha=0.80$ and $\alpha=0.70(24)$.

The Brief Sensation Seeking Scale (BSSS) (26) is an 8item scale assessing sensation seeking through four dimensions ( 2 items for each): experience seeking, adventure and thrill seeking, susceptibility, and boredom and disinhibition. Each item is rated on a 5-point scale, ranged from strongly disagree to strongly agree with total scores varying from 0 to 32 (e.g., I prefer friends who are exciting and unpredictable). The higher the score, the more the participant can be considered impulsive. Cronbach's alpha of the original scale is $\alpha=0.76$

Borderline personality traits were assessed with the Borderline Personality Scale of the Personality Diagnostic Questionnaire (PDQ-4 +) (27). Its 9 items were rated on a 7-point scale from not true at all to totally true (e.g., I'll go to extremes to prevent those who I love from ever leaving 
me). Total scores vary from 9 to 63, with higher scores suggesting a higher borderline symptomatology.

\subsubsection{Interpersonal Variables}

We used the Parental Bonding Instrument (PBI) (28) to explore participants' perceptions about their relationship with their mother and assess maternal style through perceived care (12 items) and perceived overprotection (13 items). Each item is rated on a 3-point scale ranging from almost never to almost always (e.g., was affectionate to $\mathrm{me})$. Scores range from 0 to 36 for perceived care and from 0 to 39 for overprotection, with cutoff scores of 27 and 13.5. Four types of parental styles can be distinguished: neglectful parenting (i.e., low care and low overprotection), optimal parenting (i.e., high care and low overprotection), affectionless control (i.e., low care and high overprotection) and affectionate constraint (i.e., low care and low overprotection). Cronbach's alphas are $\alpha=0.89$ for care dimension and $\alpha=0.68$ for overprotection dimension ( $\alpha=0.81$ for the total scale) (28).

We also assessed attachment with a short form of the Inventory of Parent and Peer Attachment (IPPA-24) (29). This scale is divided into two equal sections (i.e., parents/peers), each exploring three dimensions: trust, communication, and separation (4 items each). Items are rated on a 4-point scale ranged from almost never to almost always (e.g., My mother respects my feelings). Total scores range between 12 and 48 for each section. A score higher than 36 suggests a high degree of parent attachment. Scores higher than 26 for men and 32 for women indicate a high degree of peer attachment. Cronbach's alphas are $\alpha=0.82$ for parent attachment and $\alpha=0.80$ for peer attachment (29).

\subsection{Data Analyses}

Descriptive statistics were reported for all variables of interest (Table 1). We conducted (Pearson) correlational analysis to explore the relationship between PFU symptoms and studied variables. An exploratory factor analysis (EFA) was performed on the PFU scale, using the latent root criterion for retaining factors with eigenvalues greater than 1.0 and the scree plot. A hierarchical multiple regression analysis was conducted to evaluate the contribution of studied variables to PFU score in the total sample, with gender as a covariate and. As it was significant, this analysis was performed in both genders. Finally, to test for the mediation effects of overprotection and borderline traits, four regression analyses were conducted. Cronbach's alphas were explored and reported (Table1). All analyses were conducted using SPSS 21.

\section{Results}

\subsection{Reliability and Validity of the PFU Scale}

The PFU scale achieved an excellent reliability, with a Cronbach's alpha of $\alpha=0.86$. Exploratory factor analysis yielded one factor with an eigenvalue above 1 and factor loadings greater than 0.3 . This factor explained $28 \%$ of the total variance. Construct validity was assessed through associations of the total score with time spent on Facebook: Pearson's coefficient was $\mathrm{r}=0.43, \mathrm{P}<0.001$.

\subsection{Prevalence of PFU}

The prevalence of PFU was $10 \%(n=47)$, with $12 \%$ of females $(n=42)$ and $5 \%$ of males $(n=5),(P=0.02)$, scoring higher than 49. No participants had a severe problematic use (scored higher than 79). Females had higher scores of PFU than males (36.5 \pm 9.8 vs. $33.4 \pm 8, \mathrm{t}=2.9, \mathrm{P}=0.003$ ).

\subsection{Correlations}

Among our sample, PFU scores were significantly related to motives (between $r=0.30$ and $r=0.52$ ), borderline traits $(r=0.32)$, depressive symptoms $(r=0.31)$, anxiety $(r=$ $0.30)$, perceived overprotection $(r=0.18)$, perceived care $(r$ $=-0.16)$, parent attachment $(\mathrm{r}=-0.16) \mathrm{P}<0.01$, and peer attachment $(\mathrm{r}=-0.10, \mathrm{P}<0.05)$. PFU scores were not related to sensation seeking $(\mathrm{r}=0.046, \mathrm{P}=0.33)$.

\subsection{Multiple Regression Analysis Predicting PFU Symptoms}

In the first step, interpersonal variables accounted for $4 \%$ of the variance in PFU score among both gender groups. Maternal overprotection was the only significant predictor among females $(\beta=0.12, \mathrm{t}=2.0, \mathrm{P}=0.04)$ and males $(\beta=$ $0.24, \mathrm{t}=2.1, \mathrm{P}=0.03)$. Motives were entered in the second step and accounted for an increase of $34 \%$ in explained variance among females and $36 \%$ among males. Passing time was the only significant predictor in males $(\beta=0.45, \mathrm{t}=$ $4.6, \mathrm{P}=0.000)$. It was also significant among females $(\beta$ $=0.27, \mathrm{t}=5.0, \mathrm{P}=0.000)$, as were virtual community $(\beta=$ $0.33, \mathrm{t}=5.4, \mathrm{P}=0.000)$ and entertainment $(\beta=0.13, \mathrm{t}=2.0$, $\mathrm{P}=0.03$ ). Psychopathological variables were entered in the third step, for an additional $2 \%$ of variance among females. Borderline personality traits were a significant predictor among females $(\beta=0.15, \mathrm{t}=2.3, \mathrm{P}=0.02)$.

\subsection{Tests of Mediation Effects}

Among females, overprotection $\left(\mathrm{R}^{2}=0.09, \beta=0.55, \mathrm{t}\right.$ $=6.0, \mathrm{P}<0.0001)$ was a significant predictor of borderline traits; both borderline traits $\left(\mathrm{R}^{2}=0.12, \beta=0.27, \mathrm{t}=7.0, \mathrm{P}\right.$ $<0.0001)$ and overprotection $\left(\mathrm{R}^{2}=0.02, \beta=0.24, \mathrm{t}=3.3\right.$, $\mathrm{P}<0.001$ ) were significant predictors of PFU symptoms; in the fourth regression equation $\left(\mathrm{R}^{2}=0.12\right)$, borderline traits 
Table 1. Descriptive Analyses $(n=456)^{a}$

\begin{tabular}{|c|c|c|c|c|}
\hline & Minimum & Maximum & Mean \pm SD & $\alpha$ \\
\hline Facebook Use & 20 & 68 & $35.7 \pm 9.4$ & 0.86 \\
\hline \multicolumn{5}{|l|}{ Facebook Motives } \\
\hline Relationship maintenance & 8 & 30 & $22.3 \pm 4.2$ & 0.57 \\
\hline Passing time & 4 & 20 & $13.3 \pm 3.9$ & 0.70 \\
\hline Virtual community & 5 & 23 & $7.9 \pm 3.4$ & 0.77 \\
\hline Entertainment & 5 & 25 & $14.9 \pm 4.6$ & 0.81 \\
\hline Coolness & 3 & 15 & $6.0 \pm 2.4$ & 0.59 \\
\hline Companionship & 3 & 15 & $4.5 \pm 2.5$ & 0.86 \\
\hline Borderline traits & 10 & 66 & $30.7 \pm 12$ & 0.84 \\
\hline Depressive symptoms & 0 & 28 & $9.1 \pm 5.5$ & 0.84 \\
\hline Social anxiety & 18 & 90 & $44.5 \pm 16.1$ & 0.94 \\
\hline Sensation seeking & 0 & 32 & $17.3 \pm 6.5$ & 0.78 \\
\hline \multicolumn{5}{|l|}{ Parental Bonding } \\
\hline Care & 0 & 36 & $27.0 \pm 7.6$ & 0.92 \\
\hline Overprotection & 0 & 36 & $8.8 \pm 6.9$ & 0.83 \\
\hline \multicolumn{5}{|l|}{ Attachment } \\
\hline Parents & 14 & 48 & $35.7 \pm 6.9$ & 0.86 \\
\hline Peers & 14 & 48 & $37.4 \pm 5.9$ & 0.84 \\
\hline
\end{tabular}

Abbreviation: SD, Standard Deviation.

a $\alpha$, Cronbach's alpha.

were a significant predictor of PFU symptoms $(\beta=0.25, \mathrm{t}=$ $6.2, \mathrm{P}<0.0001)$ while overprotection was no longer a significant predictor $(\beta=0.10, \mathrm{t}=1.3, \mathrm{P}=0.16)$, suggesting a perfect mediation.

Among males, overprotection $\left(\mathrm{R}^{2}=0.05, \beta=0.36, \mathrm{t}=\right.$ 2.6, $\mathrm{P}<0.001)$ was a significant predictor of borderline traits; both borderline traits $\left(\mathrm{R}^{2}=0.02, \beta=0.15, \mathrm{t}=2.0, \mathrm{P}\right.$ $<0.05)$ and overprotection $\left(\mathrm{R}^{2}=0.04, \beta=0.26, \mathrm{t}=2.4, \mathrm{P}<\right.$ $0.05)$ were significant predictors of PFU symptoms; in the fourth regression equation $\left(R^{2}=0.05\right)$, overprotection was a significant predictor of PFU symptoms $(\beta=0.22, \mathrm{t}=1.9, \mathrm{P}$ $<0.05)$ while borderline traits were no longer a significant predictor $(\beta=0.11, \mathrm{t}=1.5, \mathrm{P}=0.13)$, also suggesting a perfect mediation.

\section{Discussion}

This study found that a significant minority of adolescents and young adults reported PFU according to our assessment tool. The higher proportion of PFU among females is in agreement with Andreassen (6). In accordance with Sheldon, motives and particularly passing time were the main predictors of PFU in both genders (10). Among females, entertainment was also significant, in agreement with previous results (30). In our study, the impact of virtual community suggests that social motives may play a significant role in the development of PFU.

Apart from motives, the main predictor in both genders was maternal overprotection. When feeling overprotected, adolescents and young adults may need to invest in social networks to escape from the family environment. To our knowledge, this is the first study exploring the contribution of parental bonding and borderline traits to PFU. Thus, this relationship should be further studied.

Regression analyses showed that only one variable (borderline personality traits) was a significant predictor for PFU. The association between these traits and PFU symptoms was consistent with a previous study highlighting that emotional instability is a risk factor for PIU (9). Some borderline traits, such as intolerance of loneliness and heightened need for social relationships, may increase Facebook involvement. This may in turn facilitate interpersonal disinhibition and aggravate instability of interpersonal relationships and instability of identity. So the influence between borderline traits and PFU may be reciprocal. Girls, who are more socially oriented than boys (31) and 
who preferentially use social networking sites for maintaining relationships $(30,32)$, may be more sensitive to this reciprocal effect. Multiple regression analyses showed that borderline traits were a significant predictor of PFU symptoms in girls only. This result is explained by a mediation effect. Mediation analyses revealed different relations in males and females. The predominant causal directions seemed to be gender-specific. In females, borderline traits were a mediator in the relation between overprotection and PFU symptoms. Among girls, borderline traits seemed mainly a reaction to overprotection, and PFU symptoms seemed to develop in response to borderline traits and increased need of social relationships. In males, overprotection was a mediator in the relation between borderline traits and PFU symptoms. Among males, overprotection appeared as a reaction to borderline symptoms such as impulsivity and behavior problems, which are greater in males with borderline traits than in females. PFU symptoms may mainly reflect the need to escape from overprotection in males.

This study has several limitations. Mainly, its crosssectional design prevents it from inferring causal relationships. Online recruitment and self-selection may also result in sample bias.

\subsection{Conclusion}

This study suggests that maternal overprotection and borderline personality traits play a role in the development of PFU symptoms. Moreover, the relationships between overprotection, borderline traits, and PFU symptoms might be gender-specific. Therefore, further studies of PFU should investigate the role of family factors, personality, and psychopathological factors separately in males and females.

\section{Acknowledgments}

All authors read and approved the final manuscript.The authors thank all participants for completing the survey.

\section{Footnote}

Authors' Contribution: Henri Chabrol and Axelle Moreau designed the study and analyzed the data; Stephanie Laconi prepared, drafted and edited the manuscript; Manon Delfour participated in the design and carried out the data collection.

\section{References}

1. Andreassen CS, Torsheim T, Brunborg GS, Pallesen S. Development of a Facebook Addiction Scale. Psychol Rep. 2012;110(2):501-17. doi: 10.2466/02.09.18.PR0.110.2.501-517. [PubMed: 22662404].

2. Koc M, Gulyagci S. Facebook addiction among Turkish college students: the role of psychological health, demographic, and usage characteristics. Cyberpsychol Behav Soc Netw. 2013;16(4):279-84. doi: 10.1089/cyber.2012.0249. [PubMed: 23286695].

3. Alabi O.A survey of Facebook addiction level among selected Nigerian University undergraduates. New Media Mass Commun. 2013;10:70-80.

4. Marcial DE. Are you a Facebook addict? Measuring Facebook addiction in the Philippine University. Int Proc Econ Dev Res. 2013;66:12.

5. Moreau A, Laconi S, Delfour M, Chabrol H. Psychopathological profiles of adolescent and young adult problematic Facebook users. Comput Hum Behav. 2015;44:64-9.

6. Andreassen CS. New research about Facebook addiction University of Bergen website 2012 2015. Available from: http://www.uib.no/en/ news/36380/new-research-about-facebook-addiction.

7. Kormas G, Critselis E, Janikian M, Kafetzis D, Tsitsika A. Risk factors and psychosocial characteristics of potential problematic and problematic internet use among adolescents: a cross-sectional study. BMCPublic Health. 2011;11:595. doi:10.1186/1471-2458-11-595. [PubMed: 21794167].

8. Kuss DJ, Griffiths MD, Binder JF. Internet addiction in students: Prevalence and risk factors. Comput Hum Behav. 2013;29(3):959-66.

9. Kuss DJ, van Rooij AJ, Shorter GW, Griffiths MD, van de Mheen D. Internet addiction in adolescents: Prevalence and risk factors. Comput Hum Behav. 2013;29:1987-96.

10. Sheldon P. The relationship between unwillingness-to-communicate and students' Facebook use. J Media Psychol. 2008;20:67-75.

11. Hong FY, Huang DH, Lin HY, Chiu SL. Analysis of the psychological traits, Facebook usage, and Facebook addiction model of Taiwanese university students. Telematics Inf. 2014;31:597-606.

12. Jenkins-Guarnieri MA, Wright SL, Johnson BD. The interrelationships among attachment style, personality traits, interpersonal competency, and Facebook use. Psychol Popular Media Culture. 2013;2(2):117.

13. Laconi S, Andreoletti A, Chauchard E, Rodgers RF, Chabrol H. Utilisation problematique d'Internet, temps passé en ligne et traits de personnalite. L'Encephale. 2016;42(3):214-8.

14. Delfour M, Moreau A, Laconi S, Goutaudier N, Chabrol H. Utilisation problematique de Facebook a l'adolescence et au jeune age adulte. Neuropsychiatrie de l'Enfance et de l'Adolescence. 2015;63(4):244-9.

15. Oldmeadow JA, Quinn S, Kowert R. Attachment style, social skills, and Facebook use amongst adults. Comput Hum Behav. 2013;29:1142-9.

16. Schuppert HM, Albers CJ, Minderaa RB, Emmelkamp PM, Nauta MH. Parental rearing and psychopathology in mothers of adolescents with and without borderline personality symptoms. Child Adolesc Psychiatry Ment Health. 2012;6(1):29. doi: 10.1186/1753-2000-6-29. [PubMed: 22925148].

17. Gunderson JG, Lyons-Ruth K. BPD's interpersonal hypersensitivity phenotype: a gene-environment-developmental model.J Pers Disord. 2008;22(1):22-41. doi:10.1521/pedi.2008.22.1.22. [PubMed: 18312121].

18. Khazaal Y, Billieux J, Thorens G, Khan R, Louati Y, Scarlatti E, et al. French validation of the internet addiction test. Cyberpsychol Behav. 2008;11(6):703-6. doi: 10.1089/cpb.2007.0249. [PubMed: 18954279].

19. Young KS. Internet addiction: The emergence of a new clinical disorder. Cyber Psychol Behav. 1998;1:237-44.

20. Laconi S, Rodgers RF, Chabrol H. The measurement of Internet addiction: A critical review of existing scales and their psychometric properties. Comput Hum Behav. 2014;41:190-202.

21. Meena PS, Soni R, Jain M, Paliwal S. Social networking sites addiction and associated psychological problems among young adults: A study from North India. Sri Lanka J Psychiatry. 2015;6(1):14-6.

22. Andresen EM, Malmgren JA, Carter WB, Patrick DL. Screening for depression in well older adults: evaluation of a short form of the CES-D (Center for Epidemiologic Studies Depression Scale). Am J Prev Med. 1994;10(2):77-84. [PubMed: 8037935]. 
23. Cartierre N, Coulon N, Demerval R. [Confirmatory factor analysis of the short French version of the Center for Epidemiological Studies of Depression Scale (CES-D10) in adolescents]. Encephale. 2011;37(4):2737. doi: 10.1016/j.encep.2011.01.011. [PubMed: 21981887].

24. Inderbitzen-Nolan HM, Walters KS. Social Anxiety Scale for Adolescents: normative data and further evidence of construct validity.JClin Child Psychol. 2000;29(3):360-71. doi: 10.1207/S15374424JCCP2903_7. [PubMed: 10969420].

25. La Greca AM, Lopez N. Social anxiety among adolescents: linkages with peer relations and friendships. J Abnorm Child Psychol. 1998;26(2):83-94. [PubMed: 9634131].

26. Hoyle RH, Stephenson MT, Palmgreen P, Lorch EDL. Reliability and validity of a brief measure of sensation seeking. Pers Individ Differ. 2002;32:401-14.

27. Hyler DS, Baluch JD, Taylor RR. Intramural vesical endometriosis. A case report. J Reprod Med. 1994;39(10):832-4. [PubMed: 7837134].
28. Parker G, Tupling H, Brown LB. A parental bonding instrument. Br J Med Psychol. 1979;52:1-10.

29. Raja SN, McGee R, Stanton WR. Perceived attachments to parents and peers and psychological well-being in adolescence. $J$ Youth Adolesc. 1992;21(4):471-85. doi: 10.1007/BF01537898. [PubMed: 24263975].

30. Barker V. Older adolescents' motivations for social network site use: the influence of gender, group identity, and collective self-esteem. Cyberpsychol Behav. 2009;12(2):209-13. doi: 10.1089/cpb.2008.0228. [PubMed: 19250021].

31. Hill JP, Lynch ME, editors. The intensification of gender-related role expectations during early adolescence. 31. Springer; 1983.

32. Yang CC, Brown BB. Motives for using Facebook, patterns of Facebook activities, and late adolescents' social adjustment to college. J Youth Adolesc. 2013;42(3):403-16. doi: 10.1007/s10964-012-9836-x. [PubMed: 23076768] 